\title{
Monitoring and Improving Adherence to Tyrosine Kinase Inhibitors in Patients with Chronic Myeloid Leukemia: A Systematic Review
}

\section{Bee Kim Tan' \\ Ping Chong Bee ${ }^{2}$ \\ Siew Siang Chua' \\ Li-Chia Chen $\mathbb{D}^{3}$}

'School of Pharmacy, Faculty of Health and Medical Sciences, Taylor's University, Subang Jaya, Selangor, 47500, Malaysia;

${ }^{2}$ Department of Medicine, Faculty of Medicine, University of Malaya, Kuala Lumpur, 50603, Malaysia; ${ }^{3}$ Centre for Pharmacoepidemiology and Drug Safety, Division of Pharmacy and Optometry, School of Health Sciences, Faculty of Biology, Medicine and Health, University of Manchester, Manchester Academic Health Science Centre, Manchester, MI3 9PT, UK
Correspondence: Bee Kim Tan

School of Pharmacy, Faculty of Health and

Medical Sciences, Taylor's University,

Subang Jaya, Selangor, 47500, Malaysia

Tel +60356295000

Email beekim.tan@taylors.edu.my
Purpose: Suboptimal adherence to tyrosine kinase inhibitors (TKIs) is a widely recognized issue compromising the disease control and survival of patients with chronic myeloid leukemia (CML). A recently published review by Heiney et al reported inconclusive findings on the effects of a broad range of adherence enhancing interventions. The current systematic review aimed to identify studies that evaluated adherence-enhancing interventions implemented by healthcare professionals and determine their effect on CML patients' medication adherence and clinical outcomes.

Methods: A systematic literature search was performed in 5 databases for articles published between 2002 and 2021. Studies that compared adherence enhancing interventions implemented by healthcare professionals with a comparison group were included. Relevant data on study characteristics were extracted. Medication adherence and clinical outcomes between intervention and control arms were compared.

Results: Nine studies were included in two randomised controlled trials, four cohort studies, and three before-and-after comparison studies. All the included studies incorporated complex interventions, including intensive education or consultation with pharmacists, nurses or multidisciplinary team, in combination with one or more other strategies such as structured follow-up, written materials or video, psychotherapy, medication reminder or treatment diary, with the overall goal of monitoring and improving TKI adherence. Most (7 out of 9) studies demonstrated significantly better adherence to TKIs in the intervention group than the comparison group. The relative proportion of participants who adhered to TKIs ranged from 1.22 to 2.42 . The improvement in the rate of TKI doses taken/received ranged from $1.5 \%$ to $7.1 \%$. Only one study showed a significant association between intervention and clinical outcomes, with a $22.6 \%$ higher major molecular response rate and improvement in 6 out of 20 subscales of health-related quality-of-life.

Conclusion: Complex interventions delivered by healthcare professionals showed improvement in adherence to TKIs in CML patients. Further studies are required to clarify the costeffectiveness of adherence-enhancing interventions.

Keywords: medication adherence, tyrosine kinase inhibitors, chronic myeloid leukemia, systematic review, complex interventions

\section{Introduction}

Chronic myeloid leukemia (CML) is a myeloproliferative neoplasm with an annual incidence of 0.7 to 1.8 per 100,000 population. ${ }^{1}$ Most CML patients are diagnosed in the chronic indolent phase; without effective treatment, the disease will progress to more advanced phases with complications from occult bone marrow failure and 
a poor prognosis. ${ }^{2}$ Over the past two decades, the prevalence of CML has risen exponentially owing to the effective treatment with tyrosine kinase inhibitors (TKI) ${ }^{1}$ that targets the BCR-ABL molecular defect of CML. Since 2001, TKIs have become the standard of care for CML, while allogeneic stem cell transplantation remains an option for patients who fail to respond adequately to TKIs. $^{2}$ A significant proportion of CML patients can now achieve deep molecular response (DMR) with TKI therapy, and some patients could even go into treatment-free remission (TFR). ${ }^{2}$

However, non-adherence to long-term TKI therapy is now a widely recognised issue in patients with CML, affecting $3 \%$ to $56 \%$ of the population. ${ }^{3}$ Suboptimal adherence to TKIs reduced CML survival, ${ }^{4}$ and increased the utilisation of healthcare resources. ${ }^{5}$ CML patients with poor adherence are unlikely to achieve DMR (one of the prerequisites for TFR) compared to those who are adherent $(0 \%$ versus $43.8 \% ; \mathrm{p}=0.002){ }^{6}$ The long-term symptom burden associated with side effects of TKI may be intolerable and disrupt patients' health-related quality of life. ${ }^{7}$ Given that the number of CML patients depending on TKI for survival continues to grow, there is an emerging need to address the issue of poor treatment adherence.

A recently published review by Heiney et al on adherence enhancing interventions in CML patients treated with TKIs had reported conflicting findings. This review included studies that evaluated a variety of adherence enhancing strategies, such as varying reimbursement policy (co-payment), different choices of TKI (prescribing practice) and variation in laboratory monitoring frequency (treatment guideline). ${ }^{8}$ In studies evaluating varying reimbursement policy, Dusetzina et $\mathrm{al}^{9}$ showed worsened TKI adherence with higher copayment in contrast to Shen et al, ${ }^{10}$ who found worsened adherence when TKI was heavily subsidised. Similarly, in studies examining the effect of different TKI choices, $\mathrm{Wu}$ et $\mathrm{al}^{11}$ found better adherence with nilotinib than dasatinib. This finding is contrary to better adherence with dasatinib than nilotinib reported by Yood et $\mathrm{al}^{12}$ and Trivedi et al. ${ }^{13}$ Besides, Latremouille-Viau et $\mathrm{al}^{14}$ reported improved TKI adherence with more frequent molecular monitoring, which could be confounded by enhanced communication with healthcare providers to help patients interpret their test results that in turn open up the discussion to manage treatment non-adherence better. Since poor TKI adherence is often a multifactorial issue, the "one size fits all" approach that addresses only a single predictor of non-adherence might not be the best strategy to resolve the adherence issue in CML patients.

Oral TKI therapy reduces CML patients' contact with their clinicians. A recent scoping review suggested that education- and counselling-based programs, particularly those that integrated monitoring or routine follow-up with a provider, may improve adherence to oral TKI therapy. ${ }^{15}$ A separate systematic review of qualitative studies showed that healthcare professionals in primary care, such as general practitioners and community pharmacists, might not be the best persons to provide advice or medication use counselling for patients with CML as they may not be familiar with the disease and its treatments. ${ }^{16}$ Outside of medical care, the community-based peer support group's effort often focuses on emotional support related to cancer diagnosis. ${ }^{17}$ While this type of support may indirectly improve treatment adherence by reducing anxiety. It does not target the significant influences of TKI non-adherence reported in the literature. ${ }^{16}$ Therefore, the lack of assistance from oncology practitioners to optimise adherence to TKI remains a significant unmet need in CML care.

This systematic review aimed to identify studies that evaluated interventions implemented by healthcare professionals to improve CML patients' medication-taking behaviour and to determine their effect on patients' adherence to TKI and clinical outcomes. This review is essential to identify the adherence enhancing intervention strategies implemented by healthcare professionals to inform the delivery of healthcare services that could help CML patients better manage their condition and medications.

\section{Method}

This review aimed to answer "What are the strategies and effects of interventions implemented by healthcare professionals to improve adherence to TKI therapy in patients with CML?". The report of this systematic review followed the Preferred Reporting Items for Systematic Review and Meta-Analyses (PRISMA) ${ }^{18}$ statement and the Synthesis Without Meta-analysis (SWiM) reporting guideline. ${ }^{19}$

\section{Search Strategy}

Five electronic databases, including PubMed, Cochrane Library (Cochrane Central Register of Controlled Trials), CINAHL, APA PsycINFO and Web of Science, were searched for relevant literature published between 
January 2002 and May 2021. The search duration was selected because TKIs was first approved for the treatment of CML in 2001. Therefore, adherence interventions were most likely implemented after the first TKI was launched in the market. The search terms comprised the Medical Subject Headings (MeSH) terms and text words relevant to the research question (Table 1), including chronic myeloid leukaemia, tyrosine kinase inhibitors, medication adherence and study design. Search terms were combined with Boolean operators ("OR", "AND" and "NOT") to form structured search strategies (Appendix 1). The search strategy was initially piloted in PubMed by BKT, and the results were discussed with LCC and CSS to validate the search terms before being adapted for other databases. The reference lists of eligible articles were also checked for relevant literature.

\section{Screening and Selection}

All search results were imported into a reference management programme (ie, EndNote), and duplications were removed. After deduplication, the title and abstract of the eligible articles were screened independently by two reviewers (BKT and $\mathrm{PCB}$ ) according to the pre-specified inclusion and exclusion criteria (Table 1).

Fully published original research that evaluated the effects of interventions provided by healthcare professionals to improve TKI adherence in patients with CML were included in this review (Table 1). Although randomised controlled trial (RCT) is the most rigorous study design to evaluate the effectiveness of an intervention, only two RCTs were identified from the pilot search. Therefore, other controlled study designs, such as cohort study and before-and-after comparison study comparing adherence enhancing interventions with a comparison (no additional adherence support), were also included in this review. Studies were excluded if they (1) recruited patients with cancers other than CML or patients with CML not taking TKI, (2) evaluated interventions (eg, medical, drug, surgical or transplant-based interventions) that were not designed to improve medication adherence; (3) did not make a comparison to the usual care; or (4) did not report TKI adherence as the outcome.

Each article was rated as either "included", "excluded" or "maybe" (title and abstract did not provide sufficient information for inclusion decision). The intraclass correlation coefficient (ICC) between reviewers was calculated using the SPSS programme ${ }^{20}$ to assess the rating consistency between the two reviewers (BKT and PCB). ${ }^{21}$ ICC values less than 0.5 , between 0.5 and 0.75 , between 0.75 and 0.9 , greater than 0.90 indicates poor, moderate reliability, good and excellent reliability, respectively. ${ }^{22}$ Both reviewers discussed the conflicts in the assigned rating until consensus was reached. The full text of all articles categorised as "relevant" and "maybe" were retrieved for further review to conclude the study selection.

\section{Quality Assessment}

The quality of included studies was assessed by two researchers (BKT and SSC) independently and then discussed to resolve any discrepancy. Three criteria were used according to the different study designs. The Cochrane risk-of-bias tool Version 2 (RoB2) was applied to assess the risk of bias in RCTs based on five domains across different aspects of the trial, including study design, conduct, and reporting. A series of signalling questions were used to categorise the judgement for each domain into "low risk of bias", "high risk of bias" or "some concern". 23 The Newcastle Ottawa quality assessment scale (NOS) was used to determine the quality of cohort studies on three dimensions with several criteria in each dimension: study group selection (4), study group comparability (1) and outcome of interest (3). A study can be awarded one star for each criterion in study group selection and outcome of interest and a maximum of two stars in study group comparability. ${ }^{24}$ The AXIS tool was used to appraise the quality of the beforeand-after comparison studies. It consists of 20 questions that evaluated several aspects of the study: introduction (1), methods (10), results (5), discussion (2) and others (2), and each question can be categorised as "yes", "no" or "do not know". 25

\section{Data Extraction and Analysis}

Data on study design, author, year of publication, country, sample size, intervention strategies and comparator, adherence measure and data sources, as well as the results on adherence and other outcomes (ie, clinical, humanistic or economic outcomes), were extracted by BKT in a standardised form and reviewed by LCC. The form was developed after a discussion between BKT and LCC and piloted on two studies to assess whether the data extraction meets the aim of this review.

Participants' adherence to TKI with and without intervention was presented in a forest plot without performing any meta-analysis of the effect size across different types of studies. This is due to the high heterogeneity in the study design, intervention strategies and adherence definition across the included studies. Comparing intervention versus no intervention groups, 
Table I Inclusion and Exclusion Criteria of Systematic Review

\begin{tabular}{|c|c|c|}
\hline Concept & Inclusion Criteria & Exclusion Criteria \\
\hline Population & $\begin{array}{l}\text { Patients with chronic myeloid leukemia taking tyrosine kinase } \\
\text { inhibitors }\end{array}$ & $\begin{array}{l}\text { Mixed cancer population other than chronic myeloid leukemia, } \\
\text { or patients with chronic myeloid leukemia but not taking } \\
\text { tyrosine kinase inhibitors }\end{array}$ \\
\hline Intervention & $\begin{array}{l}\text { Education, counselling, cognitive behavioural therapy, adherence } \\
\text { aids, mobile-based or any other interventions provided by } \\
\text { healthcare providers to monitor and improve medication } \\
\text { adherence }\end{array}$ & Medical, drug, surgical or transplantation-based intervention \\
\hline Comparison & Usual care (not receiving additional support for adherence) & No comparison group \\
\hline Outcome & $\begin{array}{l}\text { Medication adherence (as measured using the Morisky } \\
\text { Medication Adherence Scales, medication possession ratio, } \\
\text { etc.); with or without clinical (morbidity or mortality), } \\
\text { humanistic (quality-of-life or utility) or economic (cost- } \\
\text { minimisation, cost-effectiveness, cost-saving, cost-benefit } \\
\text { analysis) outcomes }\end{array}$ & No report on medication adherence \\
\hline Study design & $\begin{array}{l}\text { Randomized control trial, cohort study, comparison before and } \\
\text { after study }\end{array}$ & $\begin{array}{l}\text { Qualitative study, case report, case series, descriptive cross- } \\
\text { sectional study, case-control study, systematic review }\end{array}$ \\
\hline $\begin{array}{l}\text { Type of } \\
\text { publication }\end{array}$ & Original research & $\begin{array}{l}\text { Abstract, conference proceeding, protocol, preprint or editorial } \\
\text { article }\end{array}$ \\
\hline $\begin{array}{l}\text { Language of } \\
\text { publication }\end{array}$ & English & Non-English \\
\hline
\end{tabular}

the pooled relative ratio of the proportion of participants adhered to tyrosine kinase inhibitors was synthesized by random-effects Mantel-Haenszel method for RCTs and cohort studies. The weighted mean difference of tyrosine kinase inhibitors' doses taken or received was pooled by random-effects inverse variance methods for cohort studies, and before-and-after comparison studies. ${ }^{26}$ The clinical, humanistic and economic outcomes reported in the included studies between the intervention and comparison arms were also reported descriptively.

\section{Result}

\section{Study Selection}

The database search yielded 1126 results, of which 422 were excluded as duplicates. A further 691 were excluded through the screening of titles and abstracts, leaving 13 studies for full-text screening. There is a high consistency between the two reviewers (BKT and PCB) in record screening with the intraclass correlation coefficient of 0.883 and the $95 \%$ confidence interval $(95 \% \mathrm{CI})$ of 0.864 to 0.899 . Four studies were further excluded during the full-text screening, and hence, only nine relevant studies were included in the present review (Figure 1).

\section{Characteristics of Included Studies and Intervention Strategies}

The nine included studies were published from 2012 to 2021 and conducted in the United States of America (USA) $(n=3)$, Europe $(n=3)$ and Asia $(n=3)$. Various study designs were reported, including $\operatorname{RCT}^{27,28}(\mathrm{n}=2)$, cohort studies $^{29-32}(\mathrm{n}=4)$ and before-and-after comparison studies $^{32-34}(n=3)$. The majority ( 5 out of 9 ) recruited participants from multiple institutions. ${ }^{27,28,30,32,33}$ Study sample sizes ranged from 23 to 558 participants (Table 2). All included studies assessed complex interventions with several components to ensure CML patients' effective engagement and optimal adherence to TKIs.

The back-bone of the intervention comprised of intensive education and consultations with pharmacists $(\mathrm{n}=6),{ }^{27,29,30,34-36}$ nurses $(\mathrm{n}=2)^{28,32}$ or multidisciplinary team $(n=1)^{33}$ to address the barriers of poor treatment adherence. Overall, the duration of the intervention ranged from three months to five years. In both the RCTs, ${ }^{27,28}$ print materials or web-based videos were provided to supplement verbal information. Another critical component of the intervention was structured follow-up consultations to monitor and reinforce 


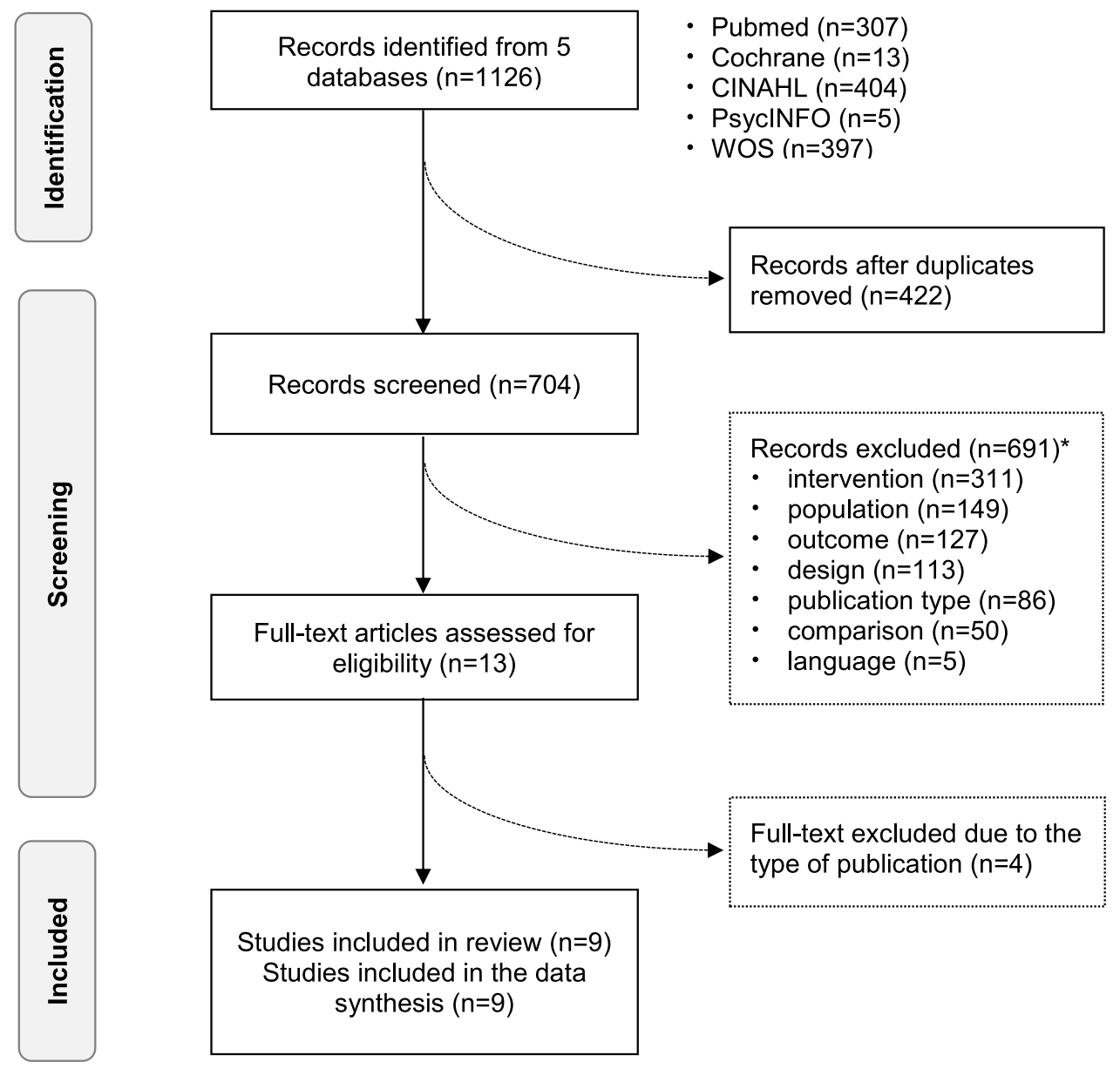

Figure I Flowchart of study selection.

Notes: PRISMA figure adapted from Page MJ, McKenzie JE, Bossuyt PM, et al. The PRISMA 2020 statement: an updated guideline for reporting systematic reviews. BMJ. 2021;372:n7I. Available from: http://creativecommons.org/licenses/by/4.0/. ${ }^{18}$

medication adherence. In general, the follow-up interval of the studies ranged from twice weekly to three times a month. In two studies, ${ }^{29,33}$ the follow-up intensity was de-escalated based on individual participants' adherence status.

Besides regular follow-up, three studies offered additional supports, including psychotherapy ${ }^{33}$ and practical reminder strategies, ie, mobile-based apps ${ }^{27}$ or short message service (SMS) text messaging. ${ }^{28,32}$ Although counselling was conducted face-to-face or via telephone call and email in most studies, one study utilized a digital approach to coach patients in self-managing their prescribed TKI therapy via bi-directional SMS interaction with a pharmacist. ${ }^{30}$ Another strategy was to use a treatment diary as a symptom and treatment tracking tool to empower patients to actively participate in managing their conditions and medications. ${ }^{34}$

\section{Quality of Included Studies}

The quality of methodology was rated as high to moderate for the two included RCTs (Table 3). Nevertheless, blinding of investigators and patients was not performed in both RCTs due to the nature of the adherence intervention. In the RCT by Kekale et al, three out of the other four criteria were not met due to the lack of description on the mechanism of allocation concealment, high dropout rate and the potentially biased selfreporting adherence. ${ }^{28}$

Similarly, the overall quality of the four cohort studies was high to moderate (Table 4). The study by Sawicki et $\mathrm{al}^{30}$ met all nine quality criteria, but the other three studies violated 1 to 3 out of the nine criteria. The study by Moon et al was rated with the lowest methodological quality due to the possibility of enrolling highly motivated patients who joined the 


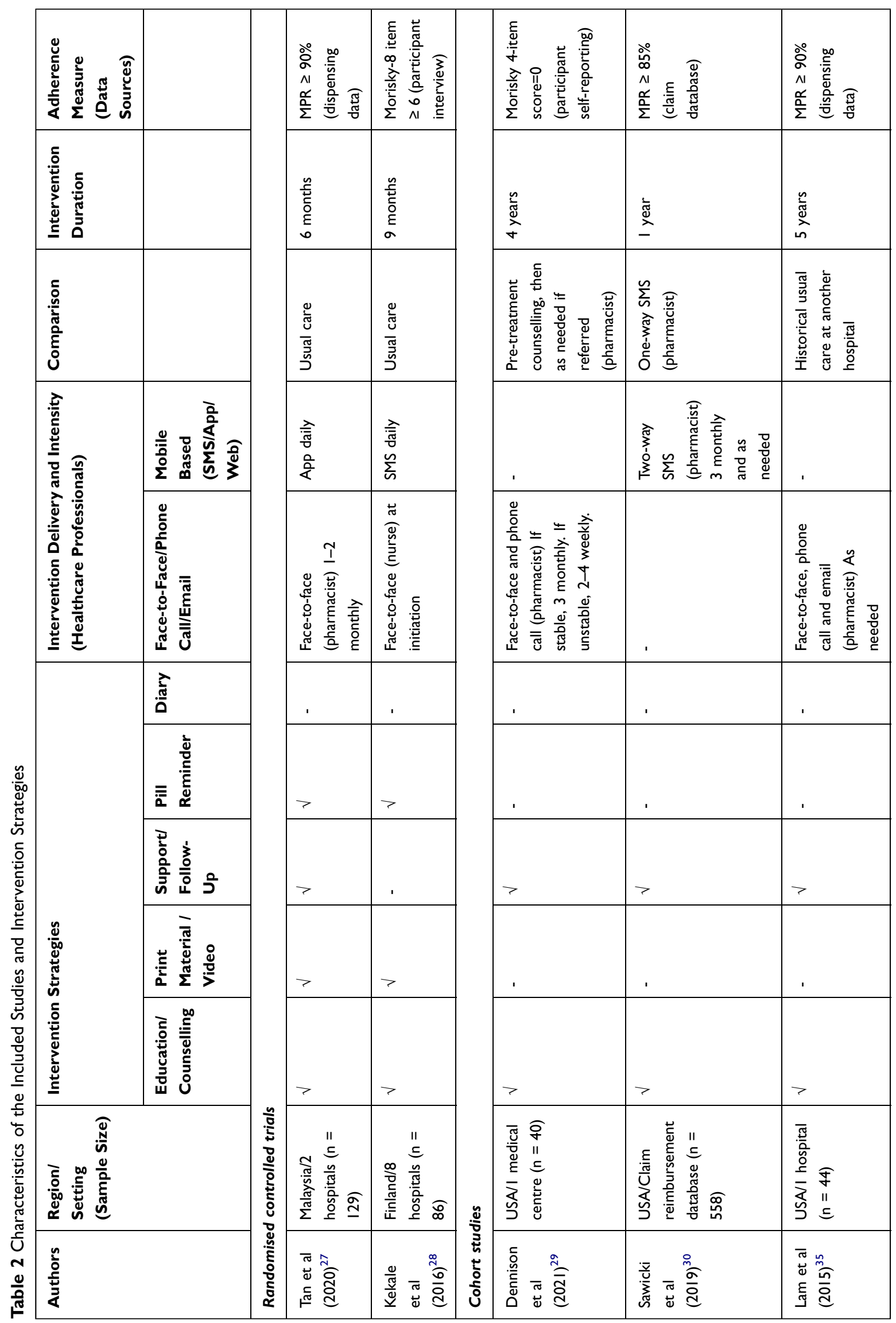




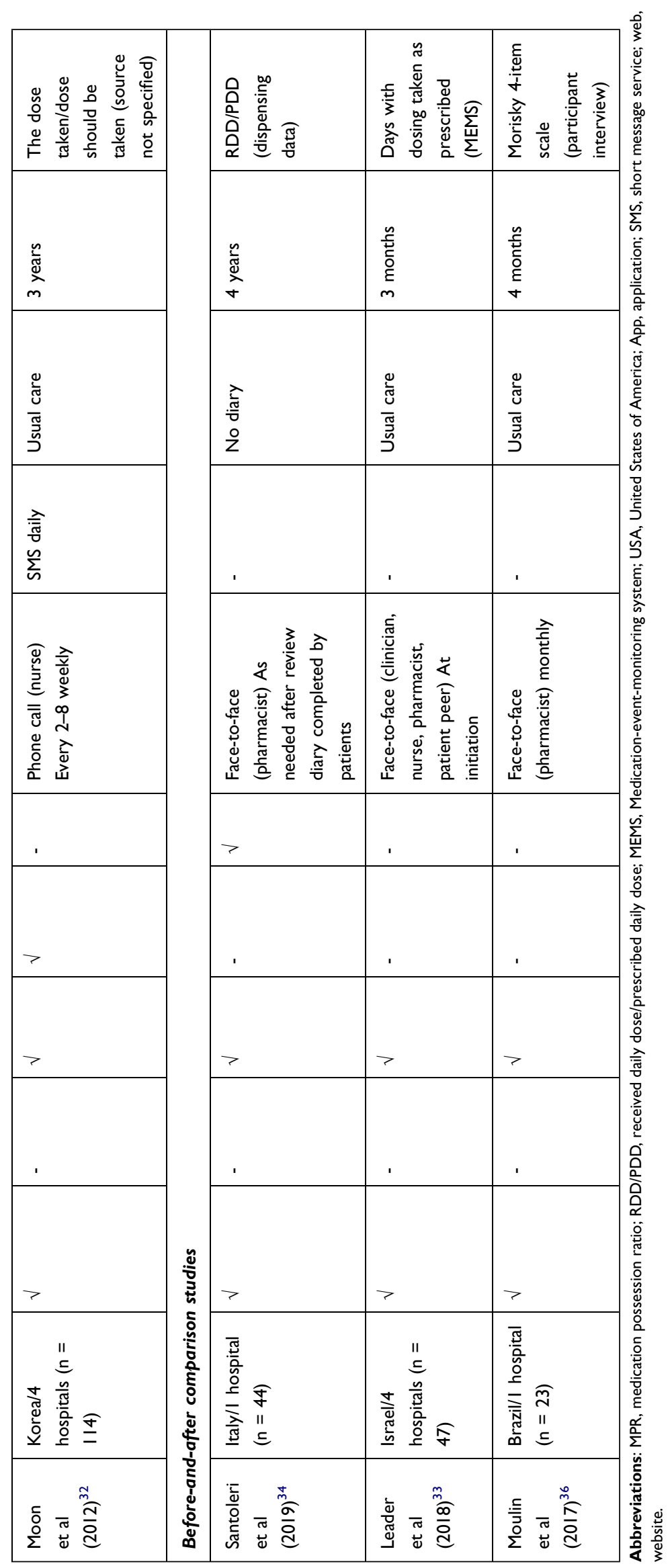


Table 3 Methodological Quality of Included Randomized Controlled Trials

\begin{tabular}{|c|c|c|c|c|c|c|}
\hline Study & $\begin{array}{l}\text { Randomization } \\
\text { Process }\end{array}$ & $\begin{array}{l}\text { Deviation from } \\
\text { Intended } \\
\text { Intervention }\end{array}$ & $\begin{array}{l}\text { Missing } \\
\text { Outcome } \\
\text { Data }\end{array}$ & $\begin{array}{l}\text { Measurement of } \\
\text { the Outcome }\end{array}$ & $\begin{array}{l}\text { Selection of the } \\
\text { Reported Result }\end{array}$ & $\begin{array}{l}\text { Overall } \\
\text { Risk }\end{array}$ \\
\hline Tan et al (2020) & + & $!$ & + & + & + & + \\
\hline Kekale et al (20I6) & $!$ & $!$ & $!$ & - & + & $!$ \\
\hline
\end{tabular}

Notes: +: low risk of bias; !: some concern; -: high risk of bias.

'Happy club" into the intervention group. In addition, the methods of assessing adherence were not specified, and the dropout rate was high. ${ }^{32}$ Additionally, the quality of the three before-and-after comparison studies (Table 5) was poor mainly due to the small sample size and short duration of follow-up (3 to 4 months $\left.{ }^{33,36}\right)$. Consequently, the long-term effect of nonrandomised interventions is unknown.

\section{Adherence Definition, Measures and Data Source}

Three approaches were used to measure TKI adherence in the nine studies (Table 2), including: (1) participants' response to questionnaire ${ }^{28,29,36}$ ) via researcher interview or participants' self-reporting, (2) electronic compilation of participants' dosing history, ie medication event monitoring system (MEMS) $)^{33}$ and (3) analysis of prescription refill data obtained from hospital dispensing record ${ }^{27,31,34}$ or claim database. ${ }^{30}$ Depending on these different measurements and data sources, various metrics were applied to quantify the adherence to TKIs. Medication possession ratio (MPR) and received/prescribed daily dose (RDD/ PDD) were used to analyze prescription refill data. Another formula, ie, "the days with dosing taken as prescribed", was used to calculate TKI adherence from the MEMS printout. One study defined TKI adherence as "the dose taken over the dose should be taken"; however, the data source for this measure was not specified. ${ }^{32}$

Overall, the nine included studies used two different constructs to compare medication adherence with and without intervention: " the relative proportion of participants who adhered to TKI" and "the rate of TKI dose taken/received". Studies that reported the relative proportion of participants who adhered to TKI used two different cut-off levels, ie, MPR $\geq 90 \%{ }^{27,35}$ or MPR $\geq 85 \% .{ }^{30}$ In the three studies using the questionnaire, optimal adherence was defined as a total score of $\geq 6$ or $\leq 0 .{ }^{28,29,36}$

\section{Association of the Intervention with TKI Adherence}

Overall, seven out of nine studies demonstrated that adherence to TKIs in the intervention group was significantly better than in the comparison group. The relative proportion of participants who adhered to TKIs ranged from 1.22 to 2.42 (Figure 2). The improvement in the rate of TKI doses taken/received ranged from $1.5 \%$ to $7.1 \%$ (Figure 3).

One cohort study in the United States by Dennison et al compared a structured pharmacist-led oral chemotherapy program to unstructured pharmacist-led counselling and found no significant difference, with a relative proportion of participants who adhered to TKIs (Rate Ratio: 0.92; 95\% CI: $0.54,1.56) .{ }^{29}$ Another cohort study in Korea by Moon et al, which implemented phone consultation with a nurse in tandem with daily medication reminder text messages, also reported a no significant change in the rate of TKI doses taken (mean difference: $-0.10 \%$; $95 \%$ CI: $-0.34 \%, 0.14 \%)^{32}$

\section{Association of Intervention with Clinical, Humanistic and Economic Outcomes}

Three out of the nine studies (including RCT, cohort study and before-and-after comparison study) reported clinical and humanistic impact of the adherence interventions. ${ }^{27,29,36}$ In contrast, none of the included studies assessed economic outcomes.

The RCT in Malaysia by Tan et al reported a statistically higher major molecular response (MMR) rate of $22.6 \%$ at the 6th month in the intervention group compared to the control group $(58.5 \%$ vs $35.9 \%$; $\mathrm{p}=$ 0.010 ) and a significantly higher DMR rate of $13.7 \%$ at 12th months $(24.6 \%$ vs $10.9 \% ; \mathrm{p}=0.042) .{ }^{27}$ In addition, the generalised estimating equation (GEE) model was used to account for confounding variables, such as participants' tyrosine kinase mutation status. The study reported a significant association between intervention and MMR 

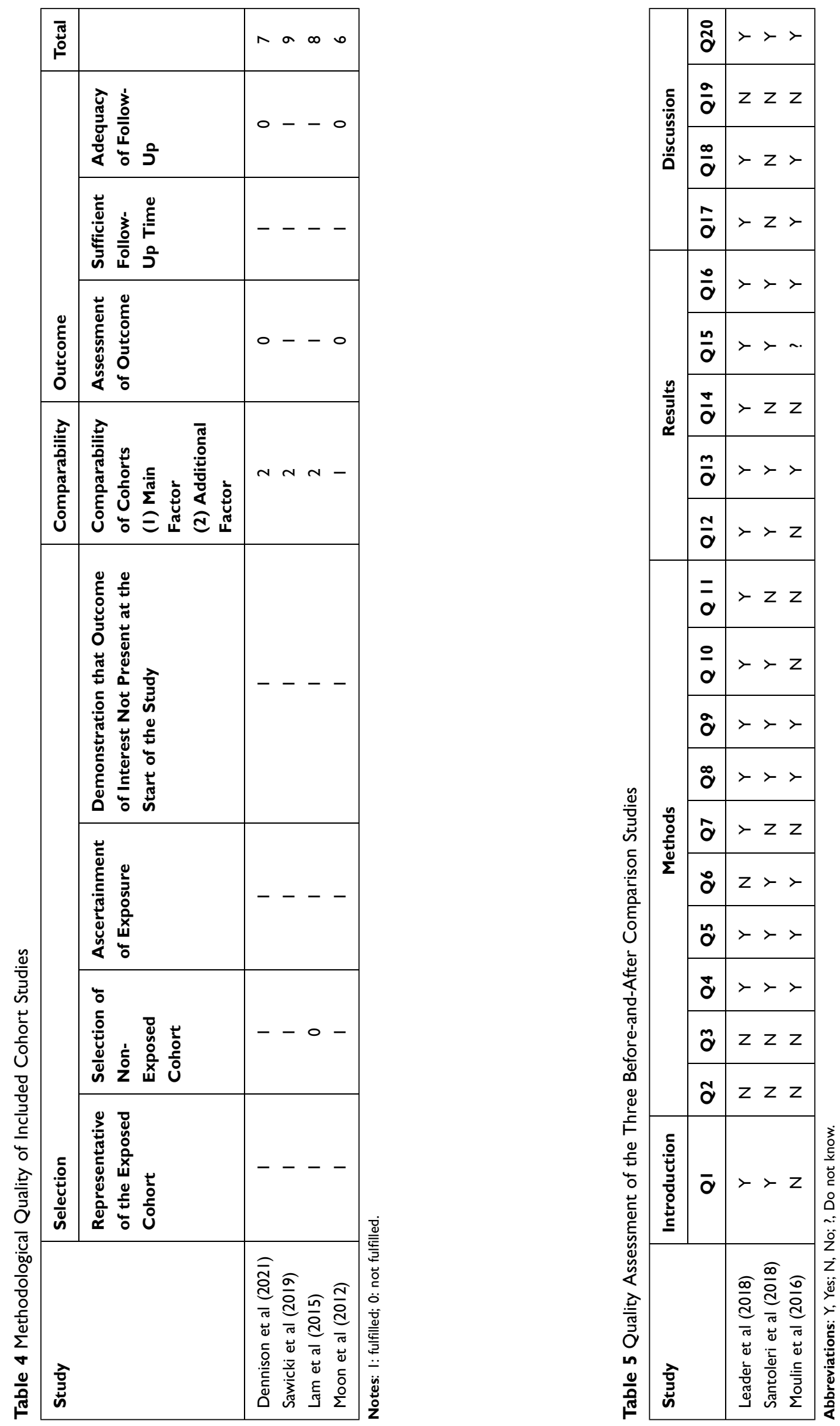


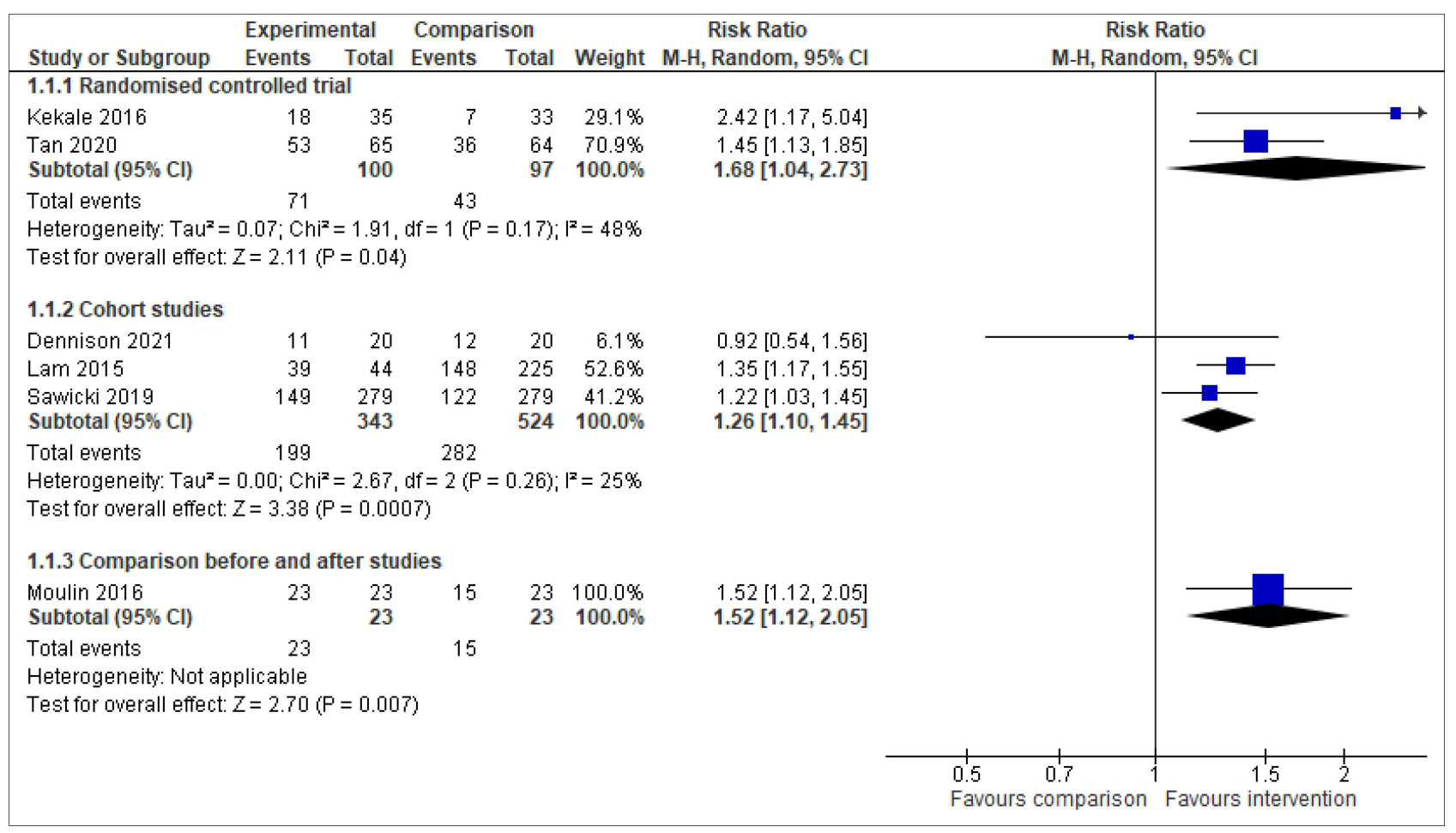

Figure 2 Relative ratio of the proportion of participants who adhered to tyrosine kinase inhibitors comparing the intervention versus no intervention groups.

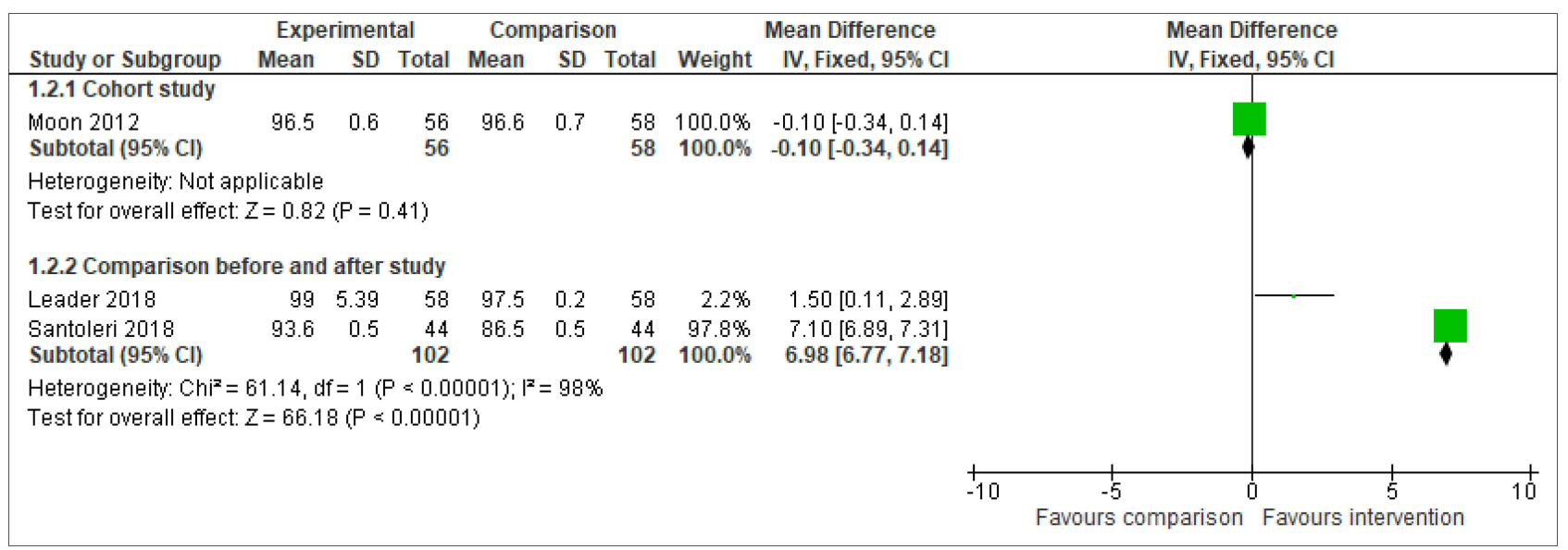

Figure 3 Mean difference of tyrosine kinase inhibitors' doses taken or received comparing intervention versus no intervention groups.

rate (odds ratio: $1.225 ; 95 \%$ CI: $1.198,1.941$ ) and DMR rate (odds ratio: $1.036 ; 95 \%$ CI: 1.006 to 2.009 ). ${ }^{27}$ Although the before-and-after comparison study in Brazil by Moulin et al also found improvement in molecular remission rate of $35 \%$ post-intervention $(95.6 \%$ vs $87 \%$ ), the statistical inference was not performed due to small sample size $(n=23) .{ }^{36}$ On the other hand, the cohort study in the United States by Dennison et al found no significant differences in the early molecular response rate $(75 \%$ vs
$75 \%, \mathrm{p}=1.000)$ and $\mathrm{MMR}$ rate $(85 \%$ vs $85 \%, \mathrm{p}=1.000)$ between the experimental and comparison groups. ${ }^{29}$

In terms of humanistic outcomes, the RCT by Tan et al showed that six out of 20 subscales of the health-related quality-of-life score were assessed using the European Organisation for Research and Treatment in Cancer (EORTC_QLQ30_CML24) questionnaire were significantly better in the intervention compared to the control arm. These included cognitive functioning $(+8.89, \mathrm{p}=$ 
$0.019)$, insomnia $(-5.56, \mathrm{p}=0.033)$, financial difficulties $(-15.55, \mathrm{p}=0.029)$, impact on worry/mood $(-9.59, \mathrm{p}=$ $0.013)$, satisfaction with care and information $(+26.11, \mathrm{p}<$ $0.001)$ and satisfaction with social life $(+21.11, \mathrm{p}<$ $0.001) .^{27}$ The before-and-after comparison study by Moulin et al and the cohort study by Dennison et al also found a reduction in the symptoms of TKI-related adverse reactions by $31 \%$ post-intervention $(70 \%$ vs $39 \%$, p < $0.05)^{36}$ and a higher rate of satisfaction with care among the intervention group $(100 \%$ vs $75 \%, p=0.047)$ respectively. However, the questionnaires used to assess TKI adverse reactions and patient satisfaction were not validated. $^{29}$

\section{Discussion}

The results of this review suggested that complex interventions delivered by healthcare professionals, such as pharmacists, nurses or multidisciplinary teams, could improve adherence to TKI in CML patients. In general, the adherence-enhancing strategies can be characterised by the "4E"s behaviour change' model, including four elements: "educate" (information and guidance delivered by healthcare professionals), "encourage" (support such as adherence aids or regular contact to reinforce behaviour change), "empower" (coaching to encourage selfmanagement of symptom and medication) and "execute" (action plan to ensure changes is embedded by disseminating the positive impact on health outcomes). The dynamics of these various intervention components will target a variety of barriers to TKI adherence to influence patients' medication-taking behaviour favourably.

The majority of the retained studies (7 out of 9) demonstrated significant improvement in TKI adherence in the intervention group, albeit a smaller effect size in some studies. For example, the before-and-after comparison study in Israel only found a marginal 1.5\% improvement in the TKI adherence rate. ${ }^{33}$ This is probably due to the high baseline adherence rate of $97.5 \%$ and a highly accurate adherence assessment method, MEMS. In contrast, two cohort studies in the United States and Korea reported no significant difference in adherence outcome. This observation could be attributed to patients in both the intervention and control groups of the United States study who received pharmacist clinical follow-up and counselling. ${ }^{29}$ Whereas, the study in Korea has a high baseline adherence rate of more than $96 \%$ and, hence, there is not much room for improvement. ${ }^{32}$ Future research should consider the level of adherence needed to allow a clinically meaningful quantification of the intervention effect and not necessary to implement any adherence intervention on patients with a high ceiling level of close to $100 \%$ baseline adherence rate.

It is challenging to draw a definite conclusion on the optimal strategies of the adherence enhancing intervention implemented by healthcare professionals, given the different combinations of adherence enhancing intervention strategies employed at different intensities and for different durations. Different adherence definitions further limit comparability of the study results, cut-off points and measures used. A variety of methods were used to assess medication adherence in the included studies, such as the questionnaire, prescription refill records and microelectronic dosing monitoring. Currently, there is no gold standard since each adherence assessment method has its strength and limitations. The main limitation with questionnaire measures is social desirability bias. Therefore, psychometrically validated tools should be used. Although prescription refill data analysis avoids patient manipulation for social desirability, it is impossible to verify whether patients took their medications. On the other hand, a micro-electronic monitoring system used a pill bottle with a cap that contained a microchip to precisely record the time the container was opened but not necessarily taken by the patient. It is also expensive and unlikely to have utility in routine clinical practice. ${ }^{3}$ Hence, this review observed a need to include two approaches of adherence measurements, for example, questionnaire and refill history, and to standardise the definition and measures of adherence to allow comparison of findings across studies, which agrees with the previous studies. ${ }^{3}$

Several included studies assessed the clinical and humanistic outcomes. Nevertheless, only one $\mathrm{RCT}^{27}$ demonstrated significant effects on molecular response and health-related quality-of-life with a robust analytical method (GEE) to account for the influence of confounding factors. In addition, the impact of these programs on preventing or limiting toxicities of oral TKI therapy has not been studied. Furthermore, no study evaluated the effect of adherence enhancing intervention on economic outcomes. Further research should evaluate the cost implication of the adherence enhancing interventions as the interventions may require substantial financial investment in time and human resources, which should be justified by potential cost saving from otherwise more frequent healthcare visits or hospitalisation to manage uncontrolled CML or complications from disease progression as a consequence of 
TKI treatment failure from suboptimal adherence. Therefore, it is crucial for future research to include economic evaluation to justify service funding and widespread implementation in routine clinical practice.

Only 2 out of 9 studies described the conceptual framework used to develop the adherence intervention. In designing the medication management service (MMS), Tan et $\mathrm{al}^{27}$ incorporated a phase by phase complex intervention development process as recommended by the Medical Research Council (MRC) United Kingdom (UK), ${ }^{37}$ and used the COM-B behavioural change model ${ }^{38}$ in designing the intervention strategies as well as in modelling the expected outcomes. Results of the qualitative study that preceded the RCT was reported in a separate publication. ${ }^{39}$ Similarly, Leader et al $^{33}$ designed their intervention based on a previously published conceptual framework ${ }^{40}$ drawn from a comprehensive literature review. Future studies should provide a theoretical grounding to justify the adherence-enhancing intervention design, which is essential to ensure the generalizability of the study findings and subsequent applications of the adherence-enhancing intervention strategies.

One of the limitations in the present review is that it only included literature published in English, and hence may have missed studies published in other languages. Secondly, the synthesis of the results in this review is limited by the high heterogeneity in study design, adherence enhancing intervention strategies, and adherence definition and measures that precluded a meta-analysis. Finally, there may be sources of systematic bias in this review as most of the retained studies were non-RCT. Nonetheless, this study is the first systematic review that assessed the effectiveness of adherence-enhancing intervention implemented by healthcare professionals in patients with CML. The results showed that complex interventions delivered by healthcare professionals within a personalised framework could improve adherence to TKIs and clinical outcomes. This review covered the CML patient population from diverse regions, cultures, health beliefs, health literacy levels and healthcare funding. The findings provide insights into potentially effective intervention strategies that are readily transferable to real-world applications.

\section{Conclusion}

The findings of the present systematic review demonstrated the importance and potential benefits of adherence enhancing interventions implemented by healthcare professionals among CML patients. Across the body of evidence, effects were consistent in improving TKI adherence via adherence enhancing strategies implemented by healthcare professionals, albeit only one study showed a significant association between adherence and clinical or humanistic outcomes. Further studies are required to evaluate the cost-effectiveness of adherence-enhancing interventions to provide decision-making evidence for healthcare institutions and policymakers.

\section{Disclosure}

The authors report no conflicts of interest in this work.

\section{References}

1. Hoglund M, Sandin F, Simonsson B. Epidemiology of chronic myeloid leukaemia: an update. Ann Hematol. 2015;94(Suppl 2):S241247. doi:10.1007/s00277-015-2314-2

2. Hochhaus A, Baccarani M, Silver RT, et al. European LeukemiaNet 2020 recommendations for treating chronic myeloid leukemia. Leukemia. 2020;34(4):966-984. doi:10.1038/s41375-020-0776-2

3. Noens L, Hensen M, Kucmin-Bemelmans I, Lofgren C, Gilloteau I, Vrijens B. Measurement of adherence to BCR-ABL inhibitor therapy in chronic myeloid leukemia: current situation and future challenges. Haematologica. 2014;99(3):437-447. doi:10.3324/ haematol.2012.082511

4. Chen TC, Chen LC, Huang YB, Chang CS. Imatinib adherence associated clinical outcomes of chronic myeloid leukaemia treatment in Taiwan. Int J Clin Pharm. 2014;36(1):172-181. doi:10.1007/ s11096-013-9876-7

5. Darkow T, Henk HJ, Thomas SK, et al. Treatment interruptions and non-adherence with imatinib and associated healthcare costs: a retrospective analysis among managed care patients with chronic myelogenous leukaemia. PharmacoEconomics. 2007;25(6):481-496. doi:10.2165/00019053-200725060-00004

6. Marin D, Bazeos A, Mahon FX, et al. Adherence is the critical factor for achieving molecular responses in patients with chronic myeloid leukemia who achieve complete cytogenetic responses on imatinib. J Clin Oncol. 2010;28(14):2381-2388. doi:10.1200/JCO.2009.26.3087

7. Efficace F, Baccarani M, Breccia M, et al. Health-related quality of life in chronic myeloid leukemia patients receiving long-term therapy with imatinib compared with the general population. Blood. 2011;118 (17):4554-4560. doi:10.1182/blood-2011-04-347575

8. Heiney SP, Sorrell M, Sheng J, et al. Interventions to Improve Adherence to Tyrosine Kinase Inhibitors in Chronic Myeloid Leukemia: a Systematic Review. Am J Clin Oncol. 2021;44 (6):291-298. doi:10.1097/COC.0000000000000818

9. Dusetzina SB, Winn AN, Abel GA, Huskamp HA, Keating NL. Cost sharing and adherence to tyrosine kinase inhibitors for patients with chronic myeloid leukemia. J Clin Oncol. 2014;32(4):306-311. doi:10.1200/JCO.2013.52.9123

10. Shen C, Zhao B, Liu L, Shih YT. Adherence to tyrosine kinase inhibitors among Medicare Part D beneficiaries with chronic myeloid leukemia. Cancer. 2018;124(2):364-373. doi:10.1002/cncr.31050

11. Wu EQ, Guerin A, Yu AP, Bollu VK, Guo A, Griffin JD. Retrospective real-world comparison of medical visits, costs, and adherence between nilotinib and dasatinib in chronic myeloid leukemia. Curr Med Res Opin. 2010;26(12):2861-2869. doi:10.1185/03007995.2010.533648

12. Yood MU, Oliveria SA, Cziraky M, Hirji I, Hamdan M, Davis C. Adherence to treatment with second-line therapies, dasatinib and nilotinib, in patients with chronic myeloid leukemia. Curr Med Res Opin. 2012;28(2):213-219. doi:10.1185/03007995.2011.649849 
13. Trivedi D, Landsman-Blumberg $P$, Darkow $T$, Smith $D$, McMorrow D, Mullins CD. Adherence and persistence among chronic myeloid leukemia patients during second-line tyrosine kinase inhibitor treatment. J Managed Care Specialty Pharm. 2014;20 (10):1006-1015. doi:10.18553/jmcp.2014.20.10.1006

14. Latremouille-Viau D, Guerin A, Gagnon-Sanschagrin P, Dea K, Cohen BG, Joseph GJ. Health Care Resource Utilization and Costs in Patients with Chronic Myeloid Leukemia with Better Adherence to Tyrosine Kinase Inhibitors and Increased Molecular Monitoring Frequency. J Managed Care Specialty Pharm. 2017;23(2):214-224. doi:10.18553/jmcp.2017.23.2.214

15. Rosenberg SM, Petrie KJ, Stanton AL, Ngo L, Finnerty E, Partridge AH. Interventions to Enhance Adherence to Oral Antineoplastic Agents: a Scoping Review. J Natl Cancer Inst. 2020;112(5):443-465. doi:10.1093/jnci/djz244

16. Hewison A, Atkin K, McCaughan D, et al. Experiences of living with chronic myeloid leukaemia and adhering to tyrosine kinase inhibitors: a thematic synthesis of qualitative studies. Eur J Oncol Nurs. 2020;45:101730. doi:10.1016/j.ejon.2020.101730

17. Cordova MJ, Giese-Davis J, Golant M, et al. Mood disturbance in community cancer support groups. The role of emotional suppression and fighting spirit. $J$ Psychosom Res. 2003;55(5):461-467. doi:10.1016/S0022-3999(03)00510-5

18. Page MJ, McKenzie JE, Bossuyt PM, et al. The PRISMA 2020 statement: an updated guideline for reporting systematic reviews. BMJ. 2021;372:n71.

19. Campbell M, McKenzie JE, Sowden A, et al. Synthesis without meta-analysis (SWiM) in systematic reviews: reporting guideline. BMJ. 2020;368:16890.

20. IBMCorp Ibm SJA, NY: IBM Corp. Statistics for Windows, Version 25.0; 2017.

21. Koo TK, Li MY. A Guideline of Selecting and Reporting Intraclass Correlation Coefficients for Reliability Research. J Chiropr Med. 2016;15(2):155-163. doi:10.1016/j.jcm.2016.02.012

22. Portney LG, Watkins MP. Foundations of Clinical Research: Applications to Practice. Vol. 892. Pearson/Prentice Hall Upper Saddle River, NJ; 2009.

23. Sterne JAC, Savović J, Page MJ, et al. RoB 2: a revised tool for assessing risk of bias in randomised trials. BMJ. 2019;366:14898. doi: $10.1136 / \mathrm{bmj} .14898$

24. Wells G, Shea B, O'Connell J. The Newcastle-Ottawa Scale (NOS) for Assessing The Quality of Nonrandomised Studies in Meta-analyses. Ottawa Health Res Inst Web Site. 2014;7.

25. Downes MJ, Brennan ML, Williams HC, Dean RS. Development of a critical appraisal tool to assess the quality of cross-sectional studies (AXIS). BMJ Open. 2016;6(12):e011458.

26. Deeks JJ, Higgins JP, Altman DG; Group obotCSM.Analysing data and undertaking meta-analyses. Cochrane Handbook for Systematic Reviews of Interventions; 2019. 241-284.

27. Tan BK, Chua SS, Chen LC, Chang KM, Balashanker S, Bee PC. Efficacy of a medication management service in improving adherence to tyrosine kinase inhibitors and clinical outcomes of patients with chronic myeloid leukaemia: a randomised controlled trial. Sup Care Cancer. 2020;28(7):3237-3247. doi:10.1007/s00520-019-05133-0
28. Kekale M, Soderlund T, Koskenvesa P, Talvensaari K, Airaksinen M. Impact of tailored patient education on adherence of patients with chronic myeloid leukaemia to tyrosine kinase inhibitors: a randomized multicentre intervention study. $J$ Adv Nurs. 2016;72 (9):2196-2206. doi:10.1111/jan.12978

29. Dennison T, Deal AM, Foster M, Valgus J, Muluneh B, PharmacistLed Oral A. Chemotherapy Program's Impact on Chronic Myeloid Leukemia Patient Satisfaction, Adherence, and Outcomes. J Adv Practitioner Oncol. 2021;12(2):148-157.

30. Sawicki C, Friend KE, Patel R, Polinski JM, Singh S. Two-Way Clinical Messaging in a CML Specialty Pharmacy Service Model. $J$ Managed Care Specialty Pharm. 2019;25(11):1290-1296. doi:10.18553/jmcp.2019.25.11.1290

31. Lam MS, Cheung N. Impact of oncology pharmacist-managed oral anticancer therapy in patients with chronic myelogenous leukemia. $J$ Oncol Pharmacy Pract. 2016;22(6):741-748. doi:10.1177/1078155215608523

32. Moon JH, Sohn SK, Kim SN, et al. Patient counseling program to improve the compliance to imatinib in chronic myeloid leukemia patients. Med Oncol. 2012;29(2):1179-1185. doi:10.1007/s12032-011-9926-8

33. Leader A, Benyamini N, Gafter-Gvili A, et al. Effect of Adherence-enhancing Interventions on Adherence to Tyrosine Kinase Inhibitor Treatment in Chronic Myeloid Leukemia (TAKE-IT): a Quasi-experimental Pre-Post Intervention Multicenter Pilot Study. Clin Lymphoma Myeloma Leuk. 2018;18(11):e449-e461. doi:10.1016/j.clml.2018.06.026

34. Santoleri F, Lasala R, Logreco A, Ranucci E, Costantini A. Using a treatment diary to improve the medication adherence in patients with chronic myeloid leukaemia. J Oncol Pharmacy Pract. 2019;25 (5):1035-1041. doi:10.1177/1078155218759184

35. Lam MS, Cheung N. Impact of oncology pharmacist-managed oral anticancer therapy in patients with chronic myelogenous leukemia. $J$ Oncol Pharmacy Pract. 2015;2:154.

36. Moulin SM, Eutrópio FJ, Souza JO, Busato FO, Olivieri DN, Tadokoro CE. The role of clinical pharmacists in treatment adherence: fast impact in suppression of chronic myeloid leukemia development and symptoms. Sup Care Cancer. 2017;25(3):951-955. doi:10.1007/s00520-016-3486-6

37. Craig P, Dieppe P, Macintyre S, et al. Developing and evaluating complex interventions: the new Medical Research Council guidance. BMJ. 2008;337:a1655. doi:10.1136/bmj.a1655

38. Michie S, van Stralen MM, West R. The behaviour change wheel: a new method for characterising and designing behaviour change interventions. Implement Sci. 2011;6:42. doi:10.1186/1748-5908-6-42

39. Tan BK, Tan SB, Chen LC, et al. Medication-related issues associated with adherence to long-term tyrosine kinase inhibitors for controlling chronic myeloid leukemia: a qualitative study. Patient Prefer Adherence. 2017;11:1027-1034. doi:10.2147/PPA.S132894

40. Gater A, Heron L, Abetz-Webb L, et al. Adherence to oral tyrosine kinase inhibitor therapies in chronic myeloid leukemia. Leuk Res. 2012;36(7):817-825. doi:10.1016/j.leukres.2012.01.021

\section{Publish your work in this journal}

Patient Preference and Adherence is an international, peer-reviewed, open access journal that focusing on the growing importance of patient preference and adherence throughout the therapeutic continuum. Patient satisfaction, acceptability, quality of life, compliance, persistence and their role in developing new therapeutic modalities and compounds to optimize clinical outcomes for existing disease states are major areas of interest for the journal. This journal has been accepted for indexing on PubMed Central. The manuscript management system is completely online and includes a very quick and fair peer-review system, which is all easy to use. Visit http:// www.dovepress.com/testimonials.php to read real quotes from published authors. 